\title{
ГОСПИТАЛЬНАЯ ДИАГНОСТИКА И РЕАБИЛИТАЦИЯ БОЛЬНЫХ С ПАРЕЗОМ ГОРТАНИ ПОСЛЕ СУБТОТАЛЬНОЙ СУБФАСЦИАЛЬНОЙ И ЭКСТРАФАСЦИАЛЬНОЙ РЕЗЕКЦИИ ЩИТОВИДНОЙ ЖЕЛЕЗЫ
}

\section{HOSPITAL DIAGNOSIS AND \\ REHABILITATION OF PATIENTS WITH LARYNGEAL PARESIS AFTER SUBTOTAL SUBFASCIAL AND EXTRAFASCIAL RESECTION OF THE THYROID GLAND \\ R. Zakhokhov \\ Z. Lovpache \\ T. Tlupova \\ A. Mukaev \\ D. Teuvazhukova}

Summary. Laryngeal paresis are identified and characterized by visualization of the larynx, which, along with the development of optical systems, significantly expands the possibilities of diagnostics and drawing up a rehabilitation plan; however, without voice diagnostics (perceptual, acoustic, aerodynamic and subjective) and electromyography, the clinical picture may be distorted. Atrophic changes in muscle tone correlate with the duration of their non-use. Therefore, voice therapy is in the first place among rehabilitation measures.

Keywords: laryngeal paresis, vocal cords, thyroid resection, diagnostic standards, rehabilitation, voice therapy.
Захохов Руслан Максидович

К.м.н., Кабардино-Балкарский государственный университет им. Х.М. Бербекова, Нальчик

Zakh_rus@mail.ru

Ловпаче Зарема Нурийдиновна

К.м.н., дочент, Кабардино-Балкарский государственный университет им. Х.М. Бербекова,

Нальчик

tanka70@yandex.ru

Тлупова Тамара Гумаровна

К.м.н., дочент, Кабардино-Балкарский государственный университет им. Х.М. Бербекова,

Нальчик

ttlupova@gmail.com

Мукаев Асланбек Ахмедович

Аспирант, Кабардино-Балкарский государственный университет им. Х.М. Бербекова, Нальчик aslanbek.mukaev@mail.ru

Теуважукова Диана Арсеновна

Клинический ординатор, Северо-Осетинская государственная медицинская академия, Владикавказ

di.teu.00@mail.ru

Аннотация. Парезы гортани идентифицируют и характеризуют посредством визуализации гортани, что, наряду с развитием оптических систем, существенно расширяет возможности диагностики и составления реабилитационного плана; однако, без голосовой диагностики (перцептивной, акустической, аэродинамической и субъективной) и электромиографии клиническая картина может быть искажена. Атрофические изменения мышечного тонуса коррелируют с продолжительностью их неиспользования, поэтому голосовая терапия стоит на первом месте в ряду реабилитационных мер.

Ключевые слова: парез гортани, голосовые связки, резекция щитовидной железы, стандарты диагностики, реабилитация, голосовая терапия.

нальной медицинской библиотеки, который используется для индексации статей для Medline. Однако голосовые связки состоят из двух пар мембран, с внешней оболочкой и внутренним телом. Контакт и вибрация этих двух складок генерируют звук во время фонации, а их дисфункция захватывает близлежащие структуры гортани. Таким образом, термин парез гортани более точно отражает анатомические и патофизиологические характеристики состояния. Цель данной работы - проанализировать международные золотые стандарты ди- 
агностики и реабилитация больных с парезом гортани после субтотальной субфасциальной и экстрафасциальной резекции щитовидной железы.

Парез гортани (далее - ПГ) возникает после субтотальной субфасциальной и экстрафасциальной резекции щитовидной железы из-за повреждения возвратного гортанного нерва (RLN) и является одним из самых тяжёлых осложнений данных операций. Нервные повреждения классифицируются как блокады нервной проводимости и повреждения аксонов. Блок нервной проводимости, также называемый неврапраксией, включает повреждение миелина неповрежденным аксоном. В таких случаях нервная функция обычно восстанавливается в течение 8 недель вместе с регенерацией шванновских клеток. Однако при повреждении аксона, известном как аксонотмезис, восстановление идет плохо. Тяжесть повреждений аксонов варьируется, так как они могут привести к гибели нейронов или к повторной иннервации клетки-мишени; эта вариация делает выздоровление пациентов непредсказуемым [2].

Симптомы, связанные с этим осложнением, включают охриплость голоса, утомляемость голоса, одышку, аспирацию, дисфагию и так далее. ПГ может быть временным или постоянным. Сообщаемая частота временных ПГ варьируется от 0\% до 12\%. Однако частота постоянного ПГ, как сообщается, значительно ниже от 0 до 3,5\% [15]. Благодаря решающей роли голоса и звучания в общении, пациенты с ПГ могут испытывать социальный дискомфорт вплоть до развития психологических нарушений; хорошо известно, что дисфония способствует социальной дезадаптации, тревожности и депрессии в случае затяжного течения ПГ. ПГ также увеличивает риск аспирации, что может привести к опасным для жизни состояниям, таким как аспирационная пневмония.

Сложности ранней диагностики ПГ заключаются в том, что у многих пациентов могут отсутствовать симптомы охриплости голоса, и послеоперационное ларингоскопическое обследование требуется всем пациентам после субтотальной субфасциальной и экстрафасциальной резекции щитовидной железы ввиду того, что ПГ значительно влияет на качество жизни пациента и иногда требует неотлагаемого начала вмешательства для предотвращения аспирации или раннего улучшения качества голоса. Знание обратимости изменений и времени до выздоровления имеет жизненно важное значение для принятия решения о необходимости краткосрочного или долгосрочного вмешательства. Enomoto et al. обнаружил 85\% выздоровление после операции по поводу доброкачественных заболеваний [6]. Lo et al. продемонстрировал выздоровление у 93\% пациентов с ПГ [14]. Nishida et al. сообщили о более чем 60\% выздоровлении ПГ в течение 6 месяцев после операции по поводу рака щитовидной железы [19].

Частота функционального восстановления после ПГ наблюдается в среднем у 72,2\% в первичной когорте и у $58,2 \%$ в когорте повторных операций. Средняя продолжительность выздоровления составляет 7 и 14,7 месяцев в первичной и повторной когортах, соответственно. По сравнению с существующей литературой, большинство нервов восстанавливают свою функцию в течение первых 6 месяцев после операции [20]. Однако в литературе видно отчётливую тенденцию - пациенты, перенесшие повторную операцию, имели более длительный интервал восстановления - до 26 месяцев. Аналогичный результат был обнаружен в масштабном исследовании Steurer et al., где выздоровление наблюдалось через два года [27].

Вопросы диагностики принципиально важны при ПГ: в целом, сочетание различных методов визуализации, включая ларингоскопию и стробоскопию, позволяет собирать обширную информацию [22]. Эндоскопическая визуализация может быть ограничена супраглоттальной гиперактивностью для преодоления недостаточности голосовой щели [23]. Hiramatsu et al. сообщили, что у 91\% пациентов с ПГ на трехмерной компьютерной томографии (КТ) наблюдается каудальное смещение структур, что затрудняет стандартную диагностику - в этих случаях гибкая ларингоскопия позволяет лучше визуализировать голосовые связки, чем зеркальная или жесткая ларингоскопия. Гибкая ларингоскопия облегчает обследование, которое меньше зависит от оператора и/или пациента [11].

Продолжительность голосового закрытия является решающим фактором в тяжести ПГ и может затруднять повседневную беседу, даже при отсутствии заметной глотальной щели - в этих ситуациях голосовая функция пациентов может быть упущена из виду при оценке с помощью ларингоскопии [30].

Видеостробоскопия стала золотым стандартом для оценки фонаторного закрытия голосовой щели и состояния слизистой оболочки. Sercarz et al. обследовали 2000 пациентов с нелеченным ПГ и обнаружили асимметричную волновую вибрацию слизистой оболочки во всех случаях, при этом непарализованная голосовая связка показывала большую амплитуду и скорость [25]. Wang et al. сообщили, что у всех пациентов с нелеченным Пг были обнаружены различные степени асимметрии слизистой оболочки и фазовых нарушений [29]. Харрис и Моррисон оценили полезность стробоскопии у 100 пациентов с ПГ. Они обнаружили, что достоверное изображение волновой вибрации слизистой оболочки 
может быть получено только у пациентов с небольшой голосовой щелью [8]. Другой недостаток - отсутствие согласия в литературе относительно того, какие стробоскопические параметры следует оценивать при ПГ [3].

Голосовые тесты в диагностике ПГ необходимы после лечения, чтобы поддержать разработку плана реабилитации, получить визуальную обратную связь и провести надлежащее сравнение голосовых результатов между методами лечения для пациентов с ПГ. Клиницисты должны оценивать многомерные аспекты голоса, включая различные голосовые особенности. Голосовую диагностику можно разделить на четыре категории: перцептивная, акустическая, аэродинамическая и субъективная. Основные параметры оценки голоса при общих голосовых нарушениях были предложены Европейским ларингологическим обществом (ELS) в 2011 году [4]. Они рекомендовали оценки показателей восприятия, включая оценку (G), грубость (R) и одышку (B) (GRB); акустику, включая джиттер, мерцание, диапазон основной частоты (F0) и самую мягкую интенсивность; аэродинамику с коэффициентом фонации; и субъективный опыт, используя анкетную оценку. В 2018 году комитет ELS опубликовал консенсусный отчет об основных параметрах, исследуемых при ПГ. В своем исследовании они проанализировали голосовые данные пациентов с ПГ, используя базы данных авторов [16]. На основании своих данных они предложили обязательно использовать перцепционный анализ со шкалой GRB и считают, что предпочтительнее проводить исследования дыхания и аэродинамики, используя, по крайней мере, МРТ, а использование акустического анализа следует рассматривать как необязательное.

В своем систематическом обзоре Desuter et al. coобщили, что из всех параметров МРТ наиболее часто демонстрировал значительное улучшение (90\%) после хирургического вмешательства, за которым следовали средний поток воздуха (86\%), шкала GRBAS (85\%), NHR (80\%), дрожание (74\%), мерцание (68\%).\%), VH (64\%), F0 (33\%) [5].

В 1944 году Уэддел впервые представил ларингеальную электромиографию (далее - LEMG) и предположил, что этот метод может иметь диагностическое и прогностическое значение для ПГ; с тех пор методика улучшилась и ее клиническое применение расширилось. Хотя визуальные и голосовые оценки широко используются, эти инструменты плохо различают неврологические и механические расстройства. В таких случаяx LEMG может быть полезен для подтверждения того, что нарушение подвижности имеет неврологическую основу, и для разработки плана лечения.
Хеман-Ака и Барр выявили, что на основе LEMG план лечения был изменен у 10 из 37 пациентов (27,0\%), а курс лечения был подтвержден у 12 из 37 пациентов $(32,4 \%)$, тогда как в план, первоначально определенный визуальной оценкой изменён у 15 из 37 пациентов $(40,0 \%)$ [9]. Focquet et al. оценили 61 пациента и обнаружили, что LEMG может успешно дифференцировать неврологическую причину в 85\% [7]. Наличие спонтанной активности (например, потенциал фибрилляции, миотонические разряды, фасцикуляции, положительные острые волны, сложные повторяющиеся потенциалы) и синкинезия указывает на плохой прогноз. Если LEMG показывает плохой результат, рекомендуется хирургическое лечение или инъекция в голосовые складки постоянным материалом [21]. Однако остается неясным когда результаты LEMG становятся показательными патологическая спонтанная активность, указывающая на дегенерацию аксонов, проявляется только через 10-14 дней после первоначального повреждения [28].

Реабилитация средствами вокальной терапии. Пациенты могут использовать компенсирующие маневры для улучшения качества голоса. Эти паттерны, часто называемые гиперфункциональными симптомами, являются реакцией на недостаточное закрытие голосовой щели и включают в себя сокращение контралатеральных голосовых складок и внешних мышц гортани, а также усиление выдоха. Эти манёвры могут привести к кратковременному улучшению качества голоса, но также связаны с утомляемостью голоса и болью в шее. Цель терапии - улучшить закрытие голосовой щели с использованием соответствующих компенсаторных механизмов и избежать нежелательного компенсаторного поведения, включая передне-заднее или латеральное надгортанное сужение, голос фальцетом и сокращение мышц глотки [13]. Основная цель послеоперационного ухода и реабилитационных мероприятий - уменьшить отек, максимально облегчить регенерацию нервов и уменьшить количество случаев стойкого пареза. В настоящее время голосовая терапия рекомендуется при послеоперационном парезе гортани во всех странах мира. Рекомендуется пациентам получать голосовую терапию как можно раньше, если нет противопоказаний для этого лечения и пациент желает её пройти голосовую терапию [17-18]. Согласно данным современной нейропатофизиологии, раннее вмешательство может предотвратить или отсрочить атрофию мышц. Возможная польза от голосовой терапии может быть определена после 1-2 сеансов. Несколько исследований показали, что 4-6 недель - минимальное время, необходимое пациентам для достижения устойчивого преимущества от голосовой терапии. Однако для пациентов с разрывом и/или аспирацией предпочтительны хирургические процедуры медиализации (SMP). Даже в таких условиях сочетание голо- 
совой терапии и хирургического лечения кажется лучшим подходом к лечению [12].

В настоящее время отсутствует стандартизация методов голосовой терапии и их клинической эффективности у пациентов с ПГ [26]. С целью активизации нервно-мышечного аппарата наружных и внутренних мышц гортани дополнительно проводится вибромассаж переднебоковой поверхности шеи и проекции входа нижнего гортанного нерва. Дыхательная гимнастика и вибромассаж подготавливают голосовой аппарат к фонации. В это время исчезают или уменьшаются парестезии, удлиняется речевой выдох, при ларингоскопии часто отмечается появление слабых колебаний голосовых складок. Основным этапом голосовой реабилитации является координация голосового аппарата фонопедическими упражнениями, или постановка голоса.

В дополнение к голосовой реабилитации, с начала 1990-х годов для инъекционной ларингопластики (ИЛ) у пациентов с ПГ были внедрены многочисленные материалы. Основная цель временной инъекции голосовой связки - восстановить функцию гортани в процессе восстановления нервной проводимости парализованной голосовой связки. Постоянный материал используется в случаях необратимого ПГ из-за явного повреждения нервов [1].

Первичная интраоперационная реиннервация RLN теоретически является идеальным подходом для улучшения послеоперационного прогноза. Используя эту процедуру во время операции, можно избежать ПГ. Интраоперационный нейромониторинг (ИОНМ) в Российской Федерации обычно не используется и может быть еще одной причиной увеличения частоты ПГ [10]. Поскольку в когорте пациентов с повторной операцией определенно выше частота как временного, так и постоянного паралича с более длительным периодом восстановления, ИОНМ может быть полезным дополнением в когорте пациентов после повторной операции.

На сегодняшний день исследуются и другие процедуры, способствующие реабилитации и регенерации, включая электростимуляцию. Отсутствуют данные для оценки её реальных эффектов в случае повреждения RLN.

\section{Эак^ючение}

Диагностика и реабилитация при ПГ должны быть адаптированы к индивидууму с учетом причины паралича и ожиданий пациента по поводу сроков выздоровления. Всем пациентам после операции следует проводить эндоскопическую оценку структуры и функции гортани, чтобы определить степень подвижности голосовых складок и наличие компенсационных явлений. Стробоскопия помогает оценить звуковое закрытие голосовой щели, волну слизистой оболочки и разницу в уровне между голосовыми складками. Однако врачи должны с осторожностью использовать инструменты визуализации в качестве единственного индикатора результатов. Вместо этого следует оценивать многомерные аспекты голоса. Базовый набор параметров может быть адаптирован в зависимости от способности пациента к эффективному участию, а также возможностей медицинского учреждения. Когда врачи подозревают поражение вдоль RLN показаны визуализирующие исследования. LEMG полезен для дифференциации причин ПГ, а также предоставляет прогностическую информацию с точки зрения восстановления нервной функции. По возможности, при прямых повреждениях гортанного нерва во время операции следует рассмотреть возможность немедленной реиннервации. Для пациентов с компенсированным голосом и без аспирации применимы менее инвазивные методы лечения, такие как голосовая терапия, массаж, выжидательный подход, ИЛ с временным материалом (в зависимости от потребностей пациента или профессии).

\section{ЛИТЕРАТУРА}

1. Коваленко Н.В. и др. Способ восстановления голосовой функции гортани после операций при раке щитовидной железы //Вестник Волгоградского государственного медицинского университета. — 2020.— № . 3 (75). - С. 50.

2. Шагатаева Б.А. и др. Реабилитация голоса при парезах и параличах гортани //Экспериментальная и клиническая оториноларингология. - 2020.№ . 2.- - C. 44-46.

3. Bonilha HS, Focht KL, Martin-Harris B. Rater methodology for stroboscopy: a systematic review. J Voice. 2015 Jan;29(1):101-8.

4. Dejonckere PH, Bradley P, Clemente P, Cornut G, Crevier-Buchman L, Friedrich G, et al. A basic protocol for functional assessment of voice pathology, especially for investigating the efficacy of (phonosurgical) treatments and evaluating new assessment techniques. Guideline elaborated by the Committee on Phoniatrics of the European Laryngological Society (ELS) Eur Arch Otorhinolaryngol. 2001 Feb;258(2):77-82.

5. Desuter G, Dedry M, Schaar B, van Lith-Bijl J, van Benthem PP, Sjogren EV. Voice outcome indicators for unilateral vocal fold paralysis surgery: a review of the literature. Eur Arch Otorhinolaryngol. 2018 Feb;275(2):459-68.

6. Enomoto K, Uchino $S$, Watanabe $S$, Enomoto Y, Noguchi S. Recurrent laryngeal nerve palsy during surgery for benign thyroid diseases: risk factors and outcome analysis. Surgery 2014; 155(3): 522-528. 
7. Focquet A, Pereon Y, Segura S, Ferron C, Malard 0, Espitalier F. Diagnostic and prognostic contribution of laryngeal electromyography in unilateral vocal-fold immobility in adults. Eur Ann Otorhinolaryngol Head Neck Dis. 2017 Feb;134(1):13-8

8. Harries ML, Morrison M. The role of stroboscopy in the management of a patient with a unilateral vocal fold paralysis. J Laryngol 0tol. 1996 Feb;110(2):141.

9. Heman-Ackah YD, Barr A. The value of laryngeal electromyography in the evaluation of laryngeal motion abnormalities. J Voice. 2006 Sep;20(3):452-60.

10. Higgins TS, Gupta R, Ketcham AS, Sataloff RT, Wadsworth JT, Sinacori JT. Recurrent laryngeal nerve monitoring versus identification alone on post-thyroidectomy true vocal fold palsy: a meta-analysis. Laryngoscope 2011; 121(5): 1009-1017.

11. Hiramatsu H, Tokashiki R, Nakamura M, Motohashi R, Yoshida T, Suzuki M. Characterization of arytenoid vertical displacement in unilateral vocal fold paralysis by three-dimensional computed tomography. Eur Arch Otorhinolaryngol. 2009 Jan;266(1):97-104.

12. Isshiki N. Mechanical and dynamic aspects of voice production as related to voice therapy and phonosurgery. Otolaryngol Head Neck Surg. 2000 Jun;122(6):782-93.

13. Kao YC, Chen SH, Wang YT, Chu PY, Tan CT, Chang WD. Efficacy of voice therapy for patients with early unilateral adductor vocal fold paralysis. J Voice. 2017 Sep;31(5):567-75.

14. Lo CY, Kwok KF, Yuen PW. A prospective evaluation of recurrent laryngeal nerve paralysis during thyroidectomy. Arch Surg Chic 2000; 135(2): $204-207$.

15. Marino M, Spencer H, Hohmann S, Bodenner D, Stack BC Jr (2014) Costs of outpatient thyroid surgery from the University Health System Consortium (UHC) database. Otolaryngol Head Neck Surg 150(2):762-769. https://doi.org/10.1161/01.STR.0000254594.33408.b1.

16. Mattei A, Desuter G, Roux M, Lee BJ, Louges MA, Osipenko E, et al. International consensus (ICON) on basic voice assessment for unilateral vocal fold paralysis. Eur Ann Otorhinolaryngol Head Neck Dis. 2018 Feb;135(1S): S11-5.

17. Mattioli F, Bergamini G, Alicandri-Ciufelli M, Molteni G, Luppi MP, Nizzoli F, Grammatica A, Presutti L (2011) The role of early voice therapy in the incidence of motility recovery in unilateral vocal fold paralysis. Logoped Phoniatr Vocol 36:40-47.

18. Mattioli F, Menichetti M, Bergamini G, Molteni G, Alberici MP, Luppi MP, et al. Results of early versus intermediate or delayed voice therapy in patients with unilateral vocal fold paralysis: our experience in 171 patients. JVoice. $2015 \mathrm{Jul} ; 29(4): 455-8$.

19. Nishida T, Nakao K, Hamaji M, Kamiike W, Kurozumi K, Matsuda H. Preservation of recurrent laryngeal nerve invaded by differentiated thyroid cancer. Ann Surg 1997; 226(1): 85-91.

20. Pei YC, Lu YA, Wong AMK, Chuang HF, Li HY, Fang TJ. Two trajectories of functional recovery in thyroid surgery related unilateral vocal cord paralysis. Surgery. 2020 0ct;168(4):578-585. doi: 10.1016/j.surg.2020.04.042. Epub 2020 Jun 28. PMID: 32605836.

21. Rickert SM, Childs LF, Carey BT, Murry T, Sulica L. Laryngeal electromyography for prognosis of vocal fold palsy: a meta-analysis. Laryngoscope. 2012 Jan;122(1):158-61.

22. Rosow DE, Sulica L. Laryngoscopy of vocal fold paralysis: evaluation of consistency of clinical findings. Laryngoscope. 2010 Jul;120(7):1376-82.

23. Rubin AD, Sataloff RT. Vocal fold paresis and paralysis. Otolaryngol Clin North Am. 2007 0ct;40(5):1109-31.

24. Sataloff RT, Praneetvatakul P, Heuer RJ, Hawkshaw MJ, Heman-Ackah YD, Schneider SM, et al. Laryngeal electromyography: clinical application. J Voice. 2010 Mar;24(2):228-34.

25. Sercarz JA, Berke GS, Ming Y, Gerratt BR, Natividad M. Videostroboscopy of human vocal fold paralysis. Ann Otol Rhinol Laryngol. 1992 Jul;101(7):567-77.

26. Speyer R. Effects of voice therapy: a systematic review. J Voice. 2008 Sep;22(5):565-80.

27. Steurer M, Passler C, Denk DM, Schneider B, Niederle B, Bigenzahn W. Advantages of recurrent laryngeal nerve identification in thyroidectomy and parathyroidectomy and the importance of preoperative and postoperative laryngoscopic examination in more than 1000 nerves at risk. Laryngoscope 2002; 112(1): 124-133.

28. Sulica L, Blitzer A. Electromyography and the immobile vocal fold. Otolaryngol Clin North Am. 2004 Feb;37(1):59-74.

29. Wang W, Chen D, Chen S, Li D, Li M, Xia S, et al. Laryngeal reinnervation using ansa cervicalis for thyroid surgery-related unilateral vocal fold paralysis: a longterm outcome analysis of 237 cases. PLoS One. 2011 Apr;6(4): e19128.

30. Woodson GE. Configuration of the glottis in laryngeal paralysis. I: Clinical study. Laryngoscope. 1993 Nov;103(11 Pt 1):1227-34

( ) Захохов Руслан Максидович (Zakh_rus@mail.ru ), Ловпаче Зарема Нурийдиновна ( tanka70@yandex.ru ),

Тлупова Тамара Гумаровна ( ttlupova@gmail.com ), Мукаев Асланбек Ахмедович ( aslanbek.mukaev@mail.ru ),

Теуважукова Диана Арсеновна ( di.teu.00@mail.ru ).

Журнал «Современная наука: актуальные проблемы теории и практики» 